by employing a full-time Emergency Management Coordinator-a pioneering position within Melbourne hospital systems. This role is broad, encompassing not only planning, but also the best practice research related to incident management, facilitation of site-based planning committees, promotion of linkages with local and state government bodies and other emergency service organizations, and the development and dissemination of educational and planning information to staff. This presentation outlines the development of this role, and provides an overview of current progress and challenges.

Providing services to an area of $1,335 \mathrm{~km}^{2}$, with a population of 553,000 people in Melbourne's western suburbs, Western Health employs 3,800 staff members by combining the resources of three leading hospitals and two specialized health programs: (1) an Aged Care and Rehabilitation Program (two nursing homes); and (2) a Drug and Alcohol Program. All hospitals are located within 20 minutes from Melbourne's central business district, and are within a major industrial petro-chemical producing belt for the State of Victoria, incorporating several major transport routes and an international and domestic airport.

Keywords: Australia; emergency medical coordinator; health; Melbourne; Western Health

Prebosp Disast Med 2005;20(2):s98-s99

\section{Road Traffic Injuries in Shanghai}

Z.M. Liu

Shanghai East Hospital, China

Worldwide, road traffic injury has become the primary public safety hazard. According to the World Health Organization (WHO), the total number of motor vehicles in the world is $>1$ billion, with $>80 \%$ in the developed countries and $<20 \%$ in the developing countries. In 2000 , an estimated 1.26 million people worldwide died as a result of road traffic injuries. The WHO predicts that road traffic injuries will account for about 2.3 million deaths globally per year by 2020 .

In 1978, the Chinese government began an open reform policy. Since then, the national economy has developed rapidly. In 2002, the number of motor vehicles in China totaled $79,756,763$. The average increase in the total motor vehicle production is $3.5 \%$ globally, and $26.4 \%$ in China for the same period. Meanwhile, however, road traffic incidents have increased at relative rates. In 2003, at the time of writing, road traffic incidents totaled 667,507 in China. This resulted in 494,174 injured patients and 104,372 deaths.

The population in Shanghai, at present, is nearly 20 million. There are 1.5 million motor vehicles within the city and the number has increased yearly. The density of population and vehicles is the highest of all Chinese cities. Although there are more and more subways and highways and roads are being widened, road traffic injuries have increased yearly. Statistics show there were 47,088 and 54,197 road traffic incidents in 2002 and 2003, respectively; there were 1,400 and 1,406 resulting deaths and 15,690 and 15,752 injured persons, respectively. Traffic injuries cost 30.05 and 39.72 million Yuan in each of those two years, respectively.
The emergency medical response in Shanghai involves the Shanghai Emergency Medical Service system and Emergency Medical Centers. The mean radius of the prehospital medical emergency network is about 15 kilometers. The "1-2-0" telephone system and other emergency numbers, such as the "1-1-0" police telephone number and the "1-1-9" fire telephone number activate the first-aid service system, which provides immediate feedback, and avoids spending time on unnecessary prehospital procedures. These emergency phone numbers have played an important role in emergency medical care in Shanghai.

In 1998, Shanghai East Hospital established a modern trauma care system and a Trauma and Emergency Center. In the Trauma and Emergency Center, there are various experienced specialists working in general surgery, neurosurgery, thoracic-cardiac surgery, orthopedic surgery, urological surgery, and professional enhanced intensive care units (EICUs) under the auspices of a coordinated emergency system. This system has performed successfully and should become the model in the management of road traffic injury patients, especially for patients with multiple injuries and for mass casualties.

Keywords: China; crashes; enhanced intensive care unit; emergency; injuries; public health; road; Shanghai; traffic; World Health Organization

Prebosp Disast Med 2005;20(2):s99

\section{Rapid Intervention Teams: The Mexican Experience in Prehospital Attention with Emergency Medical Technicians on Motorcycles}

S. San Martin Faur; G.Z. Sierra

Mexico

Introduction: In Mexico, increasing population and the corresponding increases in emergency response distances and need for emergency medical services have forced Mexican agencies involved in the delivery of emergency medical services to develop new and creative solutions to achieve their goals.

Methods: The Fire Department of Atizapan County (in the suburbs of Mexico City) implemented a Motorcycle Emergency Medical Technician's Team consisting of a Rapid Intervention Team with emergency medical technicians (EMTs) on motorcycles, as a complement to the already existing teams on ambulances. When an emergency call is received, an EMT on a motorcycle is dispatched immediately to the scene. The EMT arrives at the scene before the ambulance, and in that time starts the treatment of the victim with the equipment carried on board, stabilizes the patient, and provides early medical care (in the "Golden Hour"). At the beginning of the project, none of the EMTs had previous experience with this kind of team, so it was necessary to define the necessary job requirements of the participating EMTs, the proper medical and safety equipment, and the medical training and driving skills required in emergency conditions.

Results: Implementation of the Motorcycle Medical Technician's Team dramatically has reduced the arrival time of the medical care team to the scene and contributed to diminishing the morbidity and mortality of the victims. 